\title{
Carga de trabalho de enfermagem em sala de recuperação pós-anestésica: um estudo misto
}

Nursing workload in a postanesthesia recovery room: a mixed-methods study

Carga de trabajo de enfermería en una sala de recuperación postanestésica: un estudio mixto

\section{Ana Lúcia Uberti Pinheiro', Quézia Boeira da Cunha ${ }^{\mathrm{II}}$, Daiane Dal Pai ${ }^{\mathrm{III}}$ Rosângela Marion da Silva ${ }^{\mathrm{IV}}$, Suzinara Beatriz Soares de Lima ${ }^{\mathrm{V}}$, Silviamar Camponogara ${ }^{\mathrm{VI}}$}

Resumo: Objetivo: analisar a carga de trabalho de enfermagem em Sala de Recuperação Pós-Anestésica de um Hospital Universitário do Sul do Brasil. Método: abordagem metodológica mista, explanatório sequencial. Na etapa quantitativa, transversal, foi avaliada a carga de trabalho pelo Nursing Activities Score, em 209 pacientes. Na etapa qualitativa, foram realizadas entrevistas com 14 trabalhadores de enfermagem. A análise dos dados quantitativos foi realizada no SPSS Statistics 21.0. Os dados qualitativos passaram por análise de conteúdo de Bardin. Resultados: a carga de trabalho verificada foi de 57\%. Duas categorias surgiram: Características dos pacientes e situações determinantes para a carga de trabalho - as condições clínicas dos pacientes são apontadas como intensificadoras da carga de trabalho; e Organização do trabalho: características que influenciam na carga de trabalho - dimensionamento de pessoal e cooperação entre a equipe são fatores determinantes. Conclusão: a carga de trabalho é alta e os trabalhadores percebem características que a influenciam.

Descritores: Carga de trabalho; Enfermagem; Enfermagem em Pós-Anestésico; Downsizing organizacional

\footnotetext{
Abstract: Objective: analyzing the workload of nurses in a postanesthesia recovery room in a teaching hospital in the south of Brazil. Method: mixed-methods sequential explanatory design. In the quantitative, cross-sectional stage, the workload was evaluated using the Nursing Activities Score in 209 patients. In the qualitative stage, 14 nurses were interviewed. Quantitative data analysis was carried out using SPSS Statistics 21.0. Qualitative data went through Bardin's content analysis. Results: the workload found was 57\%. Two categories emerged:

I Enfermeira. Mestre em enfermagem. Universidade Federal de Santa Maria. Santa Maria, Rio Grande do Sul, Brasil. E-mail: aninhaupinheiro@yahoo.com.br. ORCID: 0000-0003-4658-1069

II Enfermeira. Mestre em enfermagem. Universidade Federal de Santa Maria. Santa Maria, Rio Grande do Sul, Brasil. E-mail: quezinhacunha@hotmail.com. ORCID: 0000-0001-7014-9343

III Enfermeira. Doutora em enfermagem. Universidade Federal do Rio Grande do Sul. Porto Alegre, Rio Grande do Sul, Brasil. E-mail: daiadalpai@yahoo.com.br. ORCID: 0000-0002-6761-0415

IV Enfermeira. Doutora em enfermagem. Universidade Federal de Santa Maria. Santa Maria, Rio Grande do Sul, Brasil. E-mail: cucasma@terra.com.br. ORCID: 0000-0003-3978-9654

v Enfermeira. Doutora em enfermagem. Universidade Federal de Santa Maria. Santa Maria, Rio Grande do Sul, Brasil. E-mail: suzibslima@yahoo.com.br. ORCID: 0000-0002-2162-8601

VI Enfermeira. Doutora em enfermagem. Universidade Federal de Santa Maria. Santa Maria, Rio Grande do Sul, Brasil. E-mail: silviaufsm@yahoo.com.br. ORCID: 0000-0001-9342-3683
} 
Carga de trabalho de enfermagem em sala de recuperação pós-anestésica: um estudo misto | 2

Characteristics of patients and situations that are determinant for workload - clinical conditions of the patients were said to intensify the workload; and Work organization: characteristics that influence workload - personnel sizing and team cooperation are determining factors. Conclusion: the overload is high, and the workers notice the features that influence it.

Descriptors: Workload; Nursing; Postanesthesia Nursing; Personnel Downsizing

Resumen: Objetivo: analizar la carga de trabajo de enfermería en la sala de recuperación post-anestésica de un hospital universitario en el sur de Brasil. Método: enfoque metodológico mixto, explicativo secuencial. En el paso cuantitativo, transversal, la carga de trabajo fue evaluada por el Nursing Activities Score, en 209 pacientes. En la etapa cualitativa, se realizaron entrevistas con 14 trabajadores de enfermería. El análisis de los datos cuantitativos se realizó en SPSS Statistics 21.0. Los datos cualitativos se sometieron al análisis de contenido de Bardin. Resultados: la carga de trabajo verificada fue del 57\%. Surgieron dos categorías: características de los pacientes y situaciones determinantes para la carga de trabajo: las condiciones clínicas de los pacientes se identifican como intensificadoras de la carga de trabajo; y Organización del trabajo: características que influyen en la carga de trabajo: el tamaño del personal y la cooperación entre el equipo son factores determinantes. Conclusión: la carga de trabajo es alta y los trabajadores perciben características que la influyen.

Palabras clave: Carga de trabajo; Enfermería; Enfermería Posanestésica; Redúccion de Personal

\section{Introdução}

Os trabalhadores da enfermagem brasileira sentem-se desgastados. Esse é um dos resultados encontrados em uma pesquisa inédita realizada pela Fundação Osvaldo Cruz (FIOCRUZ), por uma iniciativa do Conselho Federal de Enfermagem (COFEN), que apontou desgaste profissional em $66 \%$ dos entrevistados. O estudo traça o perfil da profissão e é considerado o mais amplo levantamento sobre uma categoria profissional já realizado na América Latina. ${ }^{1}$

Essa realidade também foi demonstrada em outros países. Um estudo realizado na Europa, em 12 países, revelou que dois em cada três enfermeiros apresentam sintomas de esgotamento elevados, mesmo entre aqueles com poucos anos de experiência. Os enfermeiros relataram que, muitos cuidados de enfermagem importantes não eram realizados, por falta de tempo, apesar de um terço deles trabalharem além do seu turno. ${ }^{2}$

A carga de trabalho é compreendida como o tempo despendido pela equipe de enfermagem para realizar atividades diretas ou indiretas da assistência ao paciente. A carga de 
3 | Pinheiro ALU, Cunha QB, Pai Daiane Dal, Silva RM, Lima SBS, Camponogara S

trabalho é influenciada pelo grau de dependência do paciente em relação aos cuidados, pela complexidade da doença, pela administração da instituição, pelo perfil da equipe de enfermagem, área física e rotinas estabelecidas na unidade, além de recursos tecnológicos disponíveis. ${ }^{3}$

Os enfermeiros têm buscado instrumentos que lhe deem objetividade para a mensuração da carga de trabalho em sua área de atuação. Nesse sentido, o Nursing Activities Score (NAS), instrumento traduzido, adaptado e validado transculturalmente para o português, ${ }^{4}$ é a escala de medida que tem demonstrado maior confiabilidade para avaliar a carga de trabalho em cuidados intensivos. O NAS tem sido utilizado em diversos países e instituições e é crescente o número de publicações que utilizam a escala com esta finalidade.

A Sala de Recuperação Pós-Anestésica (SRPA) é a unidade hospitalar responsável pelo atendimento dos pacientes após a realização de procedimentos anestésico-cirúrgicos. Essas unidades possuem características e organização particulares, pois o período de recuperação da anestesia é considerado crítico para o paciente, que apresenta necessidade de cuidados e vigilância constantes. Além disso, a presença de pacientes com necessidades de cuidados semiintensivos e intensivos é comum. Esses pacientes demandam inúmeras atividades relacionadas a suporte ventilatório e hemodinâmico invasivos. ${ }^{5}$

Com base no exposto, entende-se que a análise da carga de trabalho de enfermagem é um fator indispensável para um adequado provimento de pessoal, além de ser uma importante estratégia para avaliação da qualidade do cuidado e para promoção da saúde do trabalhador. Além disso, os estudos realizados com esta temática utilizam em sua maioria apenas a abordagem quantitativa, sem considerar as percepções dos sujeitos envolvidos nessa problemática, ou seja, os trabalhadores de enfermagem. Tendo isso em vista, essa investigação buscou avançar na compreensão do objeto de estudo, considerando que os resultados da abordagem quantitativa podem ser melhor interpretados com uma segunda fonte de dados 
Carga de trabalho de enfermagem em sala de recuperação pós-anestésica: um estudo misto | 4

qualitativa, o que levou à seguinte questão de pesquisa: Qual a carga de trabalho de enfermagem na Sala de Recuperação Pós-Anestésica (SRPA) de um Hospital Universitário, de acordo com o NAS e segundo a percepção dos trabalhadores de enfermagem? Assim, esta pesquisa objetivou analisar a carga de trabalho de enfermagem em Sala de Recuperação Pós-Anestésica de um Hospital Universitário (HU) do Sul do Brasil.

\section{Método}

O presente estudo caracteriza-se por uma abordagem metodológica mista, com utilização do modelo explanatório sequencial como estratégia de pesquisa. De acordo com esse modelo, os dados foram coletados em fases (sequencialmente), sendo realizado primeiramente a coleta de dados quantitativos e, posteriormente, a coleta de dados qualitativos. Os dados foram conectados por meio da análise da primeira fase de coleta, que subsidiou a realização da segunda fase, permitindo o aprofundamento na interpretação dos resultados. ${ }^{6}$

A investigação foi realizada na Sala de Recuperação Pós-Anestésica (SRPA) de um HU do Sul do Brasil. A SRPA dispõe de 10 leitos e atende a pacientes de diversas especialidades cirúrgicas. O quadro de pessoal é composto por pelo menos 01 enfermeiro exclusivo em cada turno de trabalho; e três a quatro técnicos de enfermagem, por turno, sendo os turnos da manhã e da tarde de 6 horas e o noturno de 12 horas.

Os participantes da pesquisa, na etapa quantitativa, foram 209 pacientes internados na SRPA, no período de junho e julho de 2016, caracterizando uma amostra por conveniência. Esses participantes foram selecionados para avaliação da carga de trabalho de enfermagem por meio da aplicação do NAS, no tempo em que permanecessem na unidade. Os critérios de inclusão na pesquisa foram: pacientes de qualquer sexo e idade, admitidos na SRPA após a realização de procedimento anestésico-cirúrgico, de qualquer especialidade médica, submetidos 
5 | Pinheiro ALU, Cunha QB, Pai Daiane Dal, Silva RM, Lima SBS, Camponogara S

a qualquer técnica anestésica e que permanecessem na unidade por um período mínimo de uma hora.

Os dados quantitativos foram coletados, de forma retrospectiva, por meio de consulta diária ao prontuário eletrônico e físico, dos pacientes, totalizando trinta dias de coleta de dados. O NAS pontua o tempo gasto pela enfermagem na realização dos 23 procedimentos listados no instrumento, cujos pesos variam de 1,2 a 32,0 pontos. O escore atribuído resulta da soma das pontuações dos itens que correspondem às necessidades de assistência direta e indireta de cada paciente. O escore varia de 0 a 176,8\%, e indica a porcentagem de tempo gasto por enfermeiro na assistência de enfermagem. Assim, uma pontuação igual a 100 pontos, por exemplo, significa que o paciente requereu $100 \%$ do tempo de um trabalhador de enfermagem no seu cuidado, nas últimas 24 horas. ${ }^{4}$

Os dados quantitativos foram organizados, mediante a dupla digitação independente em uma planilha eletrônica sob a forma de banco de dados, utilizando-se o programa Microsoft ${ }^{\oplus}$ Excel. Posteriormente à verificação de erros e inconsistências, a análise dos dados realizou-se por meio do programa SPSS Statistics versão 21.0 para Windows.

O teste de normalidade da distribuição das variáveis contínuas mensuradas neste estudo realizado foi o teste de Shapiro-Wilk, os dados atenderam à distribuição não normal, e foram realizados os testes não paramétricos como: Teste Qui-quadrado. Realizou-se Correlação de Pearson e, para a comparação de grupos, os testes de Mann-Whitney e Kruskal-Wallis. Os resultados foram considerados estatisticamente significativos quando $p<0,05$, ou seja, com intervalo de confiança de $95 \%$.

Também foram participantes do estudo 14 trabalhadores de enfermagem dessa unidade para obtenção dos dados qualitativos, os quais foram convidados a participar do estudo, respeitando-se uma proporcionalidade entre enfermeiro e técnico de enfermagem. Foi adotado como critério de inclusão: trabalhadores de enfermagem que tivessem pelo menos 50\% de sua 
Carga de trabalho de enfermagem em sala de recuperação pós-anestésica: um estudo misto |6

carga horária mensal de trabalho na SRPA e estivessem no setor por, pelo menos, seis meses. E como critérios de exclusão: trabalhadores que estivessem em licença ou afastamento por qualquer motivo, durante o período de coleta de dados, e que tivessem vínculo direto com o grupo de pesquisa promotor da investigação. A seleção dos participantes foi realizada mediante sorteio, e a coleta de dados foi realizada somente após o aceite, leitura e assinatura do Termo de Consentimento Livre e Esclarecido, sendo que o número final de entrevistas obedeceu ao critério de saturação teórica.

Tendo em vista o recurso metodológico utilizado, após a análise dos dados quantitativos, delineou-se um roteiro de entrevista semiestruturada com o objetivo de aprofundar os resultados analisados na primeira etapa do estudo, e dessa forma os dados foram conectados. Esse roteiro foi utilizado para a produção dos dados qualitativos. As entrevistas foram realizadas no primeiro semestre do ano de 2017 , as respostas foram gravadas em um microgravador digital - MP3 player e, posteriormente, transcritas pelo próprio pesquisador, o tempo médio das entrevistas foi de 20 minutos.

Os dados qualitativos foram submetidos à análise de conteúdo com base no referencial proposto por Bardin. Esta técnica de análise ocorre nas seguintes etapas: $1^{\circ}$ ) reunião do corpus de análise (entrevistas transcritas e anotações referentes); $2^{\circ}$ ) pré-análise: leitura flutuante dos dados coletados com o intuito de tornar operacionais e sistematizar as ideias iniciais; $3^{\circ}$ ) categorização de dados: a partir da leitura aprofundada do material de análise, buscando-se o estabelecimento de categorias e/ou subcategorias; $4^{\circ}$ ) análise interpretativa: quando as categorias serão trabalhadas com base nos autores da revisão de literatura, somando-se a interpretação de dados pelos pesquisadores. ${ }^{7}$ Para a organização dos dados, as entrevistas foram transcritas e identificadas pelas letras " $\mathrm{E}$ " para enfermeiro e " $\mathrm{T}$ ” para técnico de enfermagem, seguidas de números cardinais sequenciais, de acordo com a ordem de realização, constituindo o corpus da pesquisa. 
7 | Pinheiro ALU, Cunha QB, Pai Daiane Dal, Silva RM, Lima SBS, Camponogara S

A presente pesquisa cumpriu as determinações da Resolução n 466, de 12 de dezembro de 2012 do Conselho Nacional de Saúde. A mesma dispõe sobre os cuidados da pesquisa com Seres Humanos, e visa assegurar os direitos e deveres que dizem respeito aos participantes da pesquisa, à comunidade científica e ao Estado. Sob registro no CAAE 54605816.7.0000.5346 e número de parecer: 1.513.582, o projeto foi aprovado pelo Comitê de Ética em Pesquisa da instituição em Abril de 2016.

\section{Resultados}

Dos 209 pacientes da amostra, 56\% são do sexo masculino, aproximadamente 55\% provenientes do município de Santa Maria e, ainda, em torno de 38\% dos pacientes provenientes da Unidade de Clínica Cirúrgica da referida instituição. Com relação ao caráter dos procedimentos, observou-se um elevado número de cirurgias de urgência, sendo que 51\% das cirurgias realizadas foram desse tipo. Em $98 \%$ das internações, o desfecho foi a alta da unidade de recuperação anestésica, e 52\% dos pacientes foram classificados como ASA II. O tipo de anestesia mais realizado foi a anestesia geral, em $74 \%$ dos pacientes, a especialidade cirúrgica de maior prevalência foi a traumato-ortopedia com $24 \%$, seguida da especialidade de cirurgia geral $\operatorname{com} 17 \%$.

A média de idade dos pacientes foi 50,8 anos. A mediana do tempo de procedimento anestésico-cirúrgico foi de 100 minutos e, do tempo de permanência na SRPA foi 09h 35min. Em relação à carga de trabalho, a pontuação média do NAS durante a permanência dos pacientes foi de $57,31 \%$, indicando que cada paciente demanda, em média, 57\% do tempo de um trabalhador de enfermagem no seu cuidado.

$\mathrm{Na}$ aplicação do NAS, os resultados indicam um maior percentual nas atividades de monitorização e controle, investigações laboratoriais, medicação, suporte e cuidados aos 
Carga de trabalho de enfermagem em sala de recuperação pós-anestésica: um estudo misto $\mid 8$

familiares e pacientes, tarefas administrativas e gerenciais, suporte respiratório, suporte renal e intervenções específicas, conforme apresentados na Tabela 1.

Tabela 1 - Estatística descritiva das intervenções de enfermagem realizadas na Sala de Recuperação Pós-Anestésica de acordo com o Nursing Activities Score. Brasil - RS, 2016.

\begin{tabular}{|c|c|c|c|}
\hline Nursing Activities Score & Categorias & $\mathbf{N}$ & $\%$ \\
\hline \multicolumn{4}{|l|}{ Atividades Básicas } \\
\hline \multirow[t]{3}{*}{ 1. Monitorização e controles } & 1.a & 8 & $3,83 \%$ \\
\hline & 1.b & 190 & $90,90 \%$ \\
\hline & 1.c & 11 & $5,27 \%$ \\
\hline 2. Investigações laboratoriais & Sim & 132 & 63 lç, $16 \%$ \\
\hline 3. Medicação & $\operatorname{Sim}$ & 195 & $93,30 \%$ \\
\hline \multicolumn{4}{|l|}{ 4. Procedimentos de higiene } \\
\hline & 4.a & 30 & $14,35 \%$ \\
\hline & 4.b & 8 & $3,83 \%$ \\
\hline & 4.c & 4 & $1,92 \%$ \\
\hline & Não & 167 & $79,90 \%$ \\
\hline 5. Cuidados com drenos & $\operatorname{Sim}$ & 84 & $40,19 \%$ \\
\hline \multicolumn{4}{|l|}{ 6. Mobilização e posicionamento } \\
\hline & 6.a & 40 & $19,14 \%$ \\
\hline & $6 . b$ & 17 & $8,13 \%$ \\
\hline & 6.c & 1 & $0,48 \%$ \\
\hline & Não & 151 & $72,25 \%$ \\
\hline
\end{tabular}

7. Suporte e cuidados aos familiares e pacientes

\begin{tabular}{clll} 
& $7 . \mathrm{a}$ & 206 & $98,56 \%$ \\
& $7 . \mathrm{b}$ & 3 & $1,44 \%$ \\
\hline 8. Tarefas administrativas e gerenciais & & & \\
& $8 . \mathrm{a}$ & 1 & $0,48 \%$ \\
& $8 . \mathrm{b}$ & 207 & $99,04 \%$ \\
& $8 . \mathrm{c}$ & 1 & $0,48 \%$ \\
\hline
\end{tabular}

\begin{tabular}{llll}
\hline $\begin{array}{l}\text { Suporte Ventilatório } \\
\text { 9. Suporte respiratório }\end{array}$ & Sim & 142 & $67,94 \%$ \\
\hline $\begin{array}{l}\text { 10. Cuidado com vias aéreas artificiais } \\
\begin{array}{l}\text { 11. Tratamento para melhora função } \\
\text { pulmonar }\end{array}\end{array}$ & $\operatorname{Sim}$ & 19 & $9,09 \%$ \\
\hline $\begin{array}{l}\text { Suporte Cardiovascular } \\
\text { 12. Medicação vasoativa }\end{array}$ & Sim & 25 & $11,96 \%$ \\
\hline $\begin{array}{l}\text { 13. Reposição intravenosa de grandes } \\
\quad \text { perdas de fluidos }\end{array}$ & $\operatorname{Sim}$ & 22 & $10,53 \%$ \\
\hline $\begin{array}{l}\text { Suporte Renal } \\
\text { 17. Medida quantitativa débito urinário }\end{array}$ & $\operatorname{Sim}$ & 9 & $4,31 \%$ \\
\hline $\begin{array}{l}\text { Suporte Metabólico } \\
\text { 19. Tratamento acidose/alcalose metabólica }\end{array}$ & $\operatorname{Sim}$ & 126 & \\
\hline
\end{tabular}


9 | Pinheiro ALU, Cunha QB, Pai Daiane Dal, Silva RM, Lima SBS, Camponogara S

\begin{tabular}{|c|c|c|c|}
\hline complicada & & & \\
\hline 20. Hiperalimentação intravenosa & $\operatorname{Sim}$ & 3 & $1,44 \%$ \\
\hline \multicolumn{4}{|l|}{ Intervenções específicas } \\
\hline 23. Intervenções específicas fora da SRPA & Sim & 209 & $100 \%$ \\
\hline
\end{tabular}

Fonte: Elaborada pelos autores.

Nota: Os itens 14, 15, 16, 18, 21 e 22, não obtiveram pontuação em nenhuma observação.

Por meio do coeficiente de correlação de Pearson, identificou-se que as variáveis idade e NAS apresentaram uma correlação fraca $(r=0.28455, \mathrm{p}=<.0001)$, à medida que a idade aumenta, a carga de trabalho aumenta. Da mesma forma, o tempo de procedimento e a carga de trabalho apresentaram uma correlação fraca $(\mathrm{r}=0.22703, \mathrm{p}=0.0009)$, à medida que o tempo do procedimento anestésico-cirúrgico aumenta, a carga de trabalho de enfermagem gerada comporta-se da mesma maneira. Já a variável tempo de permanência, não demonstrou correlação com nenhuma das outras variáveis (idade, tempo de procedimento e NAS).

Comparando os dois grupos de pacientes, os que realizaram cirurgia eletiva $(\mathrm{N}=103)$ e os de urgência $(\mathrm{N}=106)$, com suas respectivas médias do NAS $(57,19 \%$ e $57,43 \%)$, constata-se que a diferença entre os grupos não obteve significância estatística ( $\mathrm{p}=0.5821)$. Da mesma forma, não foi observada diferença estatística significativa da carga de trabalho em relação ao tipo de anestesia $(\mathrm{p}=0.6964)$.

Em contrapartida, a relação entre as variáveis ASA e carga de trabalho apresentou significância estatística ( $\mathrm{p}=0.0006)$. Quanto maior o nível de classificação ASA, maior é a carga de trabalho gerada à enfermagem.

Com relação aos dados qualitativos, dos 14 trabalhadores que responderam às entrevistas, três eram do sexo masculino e 11 do sexo feminino; caracterizando-se por um grupo predominantemente composto por mulheres, sendo quatro solteiros, 10 casados, com idade entre 28 anos a 47 anos. Entre os participantes, quatro são enfermeiros e 10 técnicos de enfermagem. O tempo de serviço na instituição variou de oito meses a 16 anos. 
Após análise dos dados oriundos das entrevistas foi possível conhecer a percepção dos trabalhadores sobre a carga de trabalho a que estão expostos, emergindo duas categorias: Características dos pacientes e situações determinantes para a carga de trabalho e Organização do trabalho: características que influenciam na carga de trabalho.

\section{Características dos pacientes e situações determinantes para a carga de trabalho de enfermagem}

Quando questionados sobre o perfil do paciente atendido na SRPA, os trabalhadores de enfermagem definiram essa clientela como pacientes graves, com comorbidades associadas ou em estágio avançado da doença de base, o que gera uma maior demanda de cuidados por parte da equipe.

[...] uma alta demanda de pacientes sendo admitidos, pacientes sendo transferidos, e a nossa $S R$ aqui tem uma característica diferenciada, porque muitas vezes a gente atua como UTI [unidade de terapia intensiva]. Há pouco tempo, dos 10 pacientes 7 estavam em ventilação mecânica. Então, isso gera uma carga de trabalho maior e um estresse muito grande, porque ele chega na unidade, a gente tem que receber o paciente, olhar todos os drenos, todos os curativos, as comorbidades do paciente, sangramento no primeiro pós operatório (PO) dele, pós-cirúrgico [...] tem a dor e o manejo da dor $[\ldots](\mathrm{E} 1)$

Considerando o perfil dos pacientes atendidos neste cenário, os trabalhadores foram questionados acerca da principal característica destes pacientes, que demandam mais cuidados por parte da equipe de enfermagem, ou seja, que aumentam a carga de trabalho. A maioria significativa dos entrevistados relatou ser a instabilidade do paciente o fator principal para aumento da carga de trabalho, conforme segue:

Se estão instáveis, a gente passa em função deles. Então, geralmente, é isso, cirurgias grandes, que a gente tem bastante, que tem que estar toda hora, porque são " $n$ " coisas, drenos, isso e aquilo. E, como o típico dos pacientes são mais graves, então esses pacientes requerem bem mais. $\dot{A} s$ vezes, um de ventilação mecânica estável, a gente faz o que tem que fazer: 
11 | Pinheiro ALU, Cunha QB, Pai Daiane Dal, Silva RM, Lima SBS, Camponogara S

alternância, banho, medicação [...] só que as vezes tem uns que não estão em ventilação, só que estão instáveis [...] (T5)

Ainda no que tange à complexidade do cenário e às situações de urgência/emergência existentes, os depoentes ressaltam que, algumas delas são potenciais geradoras de sobrecarga para o trabalho de enfermagem, conforme explicitado na fala a seguir:

Tem noites que eu não consigo fazer intervalo. É muita coisa para fazer, muito curativo, muita instabilidade dos pacientes no pós-operatório. É aquele paciente, que como acontece no PS, a gente recebe e não sabe quem é esse paciente, de onde veio, mas ele está ali, com um tiro na cabeça e a gente está cuidando dele. (T8)

Assim, fica evidente que as características próprias dos pacientes em pós-operatório imediato, considerado um período crítico, associadas a uma clientela que muitas vezes apresenta condições prévias de saúde precárias, com comorbidades que levam a complicações potenciais, contribuem para uma percepção de elevada carga de trabalho para equipe de enfermagem que atua em SRPA no local do estudo.

\section{Organização do trabalho: características que influenciam na carga de trabalho de enfermagem}

Considerando o dimensionamento de pessoal como fator determinante para a carga de trabalho de enfermagem, ao serem questionados acerca da rotina de trabalho e divisão dos pacientes entre os membros da equipe, o déficit de trabalhadores foi mencionado:

Mas, assim, a gente fica em torno de três, quatro funcionários [...]. O ideal é quatro. Mas, a gente fica, às vezes, em três, no final de semana, em três, dai dá três pacientes para cada um, um fica com quatro, mas ai a gente divide, quem fica com quatro, fica com os mais leves teoricamente. Se tem gente suficiente, daí a gente divide os respiradores, um fica só com os ventiladores, os outros vão dividir os outros pacientes, mas isso depende de quantos funcionários a gente tem. (T4) 
Carga de trabalho de enfermagem em sala de recuperação pós-anestésica: um estudo misto | 12

Considerando as características organizacionais do trabalho, os entrevistados mencionaram a falta de leitos para receber os pacientes pós-cirúrgicos, como um fator determinante para o aumento da carga de trabalho no setor. O tempo de permanência dos pacientes é variável, sendo que, alguns permanecem internados além do necessário para recuperação do procedimento anestésico, devido a condições organizacionais. Desta forma, esta demanda implica em aumento da carga de trabalho de enfermagem:

[...] são leitos que a gente tem que pedir quase que pelo amor de Deus, recebam os nossos pacientes, porque se a $S R$ não libera os pacientes para $O$ terceiro, quarto ou quinto andar, o bloco fecha, porque a gente precisa dessa rotatividade. $O$ médico precisa liberar os pacientes no terceiro [terceiro andar - clínica cirúrgica] para receber os pacientes da SRPA principalmente, para o bloco poder liberar a cirurgia para poder sair. (E4)

Então, são pacientes praticamente internados, sempre tem banhos, na outra recuperação não teria [...] se funcionasse como recuperação. (T8)

Ao serem perguntados sobre fatores que poderiam minimizar a carga de trabalho a qual estão expostos, muitos trabalhadores mencionam a equipe de enfermagem como sendo cooperativa, gerando bem-estar entre os membros e amenizando as adversidades do processo de trabalho.

A equipe é boa, é ótima aqui na verdade, acho que é essa a palavra que tem que usar. É aquela coisa que te disse: todo mundo se ajuda, o ambiente é bom de trabalhar, nós somos uma equipe muito boa, não é o meu paciente, o teu paciente, o paciente é de todos. (T6)

Depreende-se que a elevada demanda de atividades, associada a outros fatores organizacionais/institucionais, contribuem para a percepção de elevada carga de trabalho no setor. O dimensionamento de pessoal torna-se imprescindível para que a carga de trabalho seja adequada entre os membros da equipe de enfermagem, que mesmo cooperativa, sente-se desgastada em diversos momentos do cotidiano de trabalho. 


\section{Discussão}

No presente estudo, considerando a amostra da etapa quantitativa deste estudo, 209 pacientes, a média de idade encontrada foi de 50,8 anos, $56 \%$ são do sexo masculino, $52 \%$ foram classificados como ASA II, o tipo de anestesia mais realizado foi a anestesia geral, em $74 \%$ dos pacientes, a especialidade cirúrgica de maior prevalência foi a traumato-ortopedia com $24 \%$, seguida da especialidade de cirurgia geral com 17\%. A mediana do tempo de permanência foi de 9 horas e 35 minutos e 98\% dos pacientes foram encaminhados às unidades de internação.

Com relação a caracterização dos participantes da etapa qualitativa, os trabalhadores de enfermagem majoritariamente pertencem ao sexo feminino, corroborando com os resultados de pesquisa realizada recentemente para identificar o perfil da enfermagem no Brasil, a qual evidenciou que o setor da saúde é historicamente feminino, principalmente devido à enfermagem. Há um avanço crescente dos homens, porém a equipe de enfermagem brasileira ainda é predominantemente feminina, com $85,1 \%$ dos trabalhadores. ${ }^{8}$

Em estudo semelhante, realizado em recuperação pós-anestésica, autoras identificaram média de idade dos pacientes de 51,57 anos, $54 \%$ eram do sexo feminino, $58,2 \%$ com classificação ASA II. A anestesia geral foi o tipo de anestesia mais realizada, em 76,1\% dos pacientes. Para as especialidades cirúrgicas, a prevalência maior foi para a cirurgia geral $24,6 \%$. A mediana de horas de permanência na unidade foi de $4,83(0,33-53,22)$ horas. A alta para unidade de internação ocorreu em $81,3 \%$ dos pacientes. ${ }^{5}$ Percebe-se, neste contexto, a convergência parcial entre os dados encontrados nos dois estudos realizados em cenário de recuperação anestésica.

Observa-se, nesta investigação, um tempo de permanência do paciente elevado, no entanto, não obteve relação estatisticamente significativa com a carga de trabalho, o que diverge de outra investigação, realizada em unidade de terapia intensiva (UTI), que avaliou os fatores associados à carga de trabalho em UTI e demonstrou que o tempo de permanência na UTI foi fator significativo para elevada carga de trabalho de enfermagem no primeiro dia de internação. ${ }^{9}$ 
Carga de trabalho de enfermagem em sala de recuperação pós-anestésica: um estudo misto | 14

Estes achados reforçam que pacientes com maior número de horas de permanência na unidade tendem a apresentar maior grau de dependência dos cuidados de enfermagem, em razão da assistência e da terapêutica que esses pacientes necessitam. ${ }^{5}$ Em contrapartida, na realidade local, muitas vezes, os pacientes permanecem na sala de recuperação pós-anestésica devido à falta de leitos hospitalares. Estudo sobre terapia intensiva cirúrgica em um hospital universitário, refere que a melhoria na reorganização dos cuidados de enfermagem minimiza a carga de trabalho e pode ter efeito positivo nas condições de saúde dos pacientes, inclusive no tempo de permanência em UTI. ${ }^{10}$

O índice de classificação ASA e a técnica anestésica de maior prevalência vêm ao encontro de estudo realizado em recuperação pós-anestésica, que indica percentuais semelhantes em relação a estas variáveis. O mesmo estudo evidencia um tempo de permanência de 4,83 (3,43 - 6,72) horas, consideravelmente inferior ao encontrado nesta investigação. ${ }^{11}$ Os resultados qualitativos da presente investigação permitem compreender essa questão de forma mais ampla, tendo em vista que os trabalhadores mencionam que a dificuldade de leitos de internação para o pós-operatório eleva o tempo de permanência do paciente na SRPA, o que por sua vez, também contribui para uma percepção de sobrecarga de trabalho.

Percentual significativo de pacientes apresentou classificação ASA II, ou seja, risco anestésico intermediário. Um estudo retrospectivo que avaliou a incidência de óbitos anestésico-cirúrgicos, nas primeiras 24 horas, em um HU, concluiu que ocorreram mais óbitos entre pacientes com classificação ASA III ou mais. ${ }^{12}$ No presente estudo, a classificação ASA apresentou correlação positiva com a carga de trabalho, quanto maior o índice ASA, maior o NAS. Esse achado também converge com os dados obtidos nas entrevistas, em que os trabalhadores mencionam que a presença de comorbidades nos pacientes é um dos fatores que aumentam a demanda por cuidados de enfermagem. 
15 | Pinheiro ALU, Cunha QB, Pai Daiane Dal, Silva RM, Lima SBS, Camponogara S

Com relação aos valores do NAS, em estudo realizado em unidade de recuperação pósanestésica, identificou-se um NAS médio de 76,2 (70,47 - 84,6) pontos (\%). ${ }^{11}$ Apesar de o NAS médio encontrado neste estudo ter sido mais baixo, $57,31 \%$, esse valor representa 13,7 horas de assistência de enfermagem por paciente nas 24 horas em média. Considerando-se a legislação vigente $^{13}$ esse número de horas corresponde a um cuidado a paciente com alta dependência/semi-crítico, sendo que a recomendação nesse caso é de um profissional para cada 2,4 pacientes. De acordo com os depoimentos dos trabalhadores, cada técnico de enfermagem é responsável por cerca de três ou quatro pacientes no seu turno de trabalho. Dessa forma, nos períodos em que a unidade está com sua lotação máxima, há indicativo de que pode haver sobrecarga de trabalho, ou seja, a equipe, nesse caso, estaria subdimensionada.

Nesse sentido, de acordo com o perfil de pacientes atendidos evidenciado nas falas dos participantes do estudo, percebe-se que a presença de comorbidades implica, muitas vezes, em instabilidade no pós-operatório, e, consequentemente, aumento da carga de trabalho, uma vez que estes pacientes necessitam de avaliação rigorosa de sinais vitais, parâmetros hemodinâmicos e respiratórios, a fim de evitar complicações relacionadas ao procedimento anestésico-cirúrgico. Os dados do NAS estão em consonância aos depoimentos na medida em que os itens mais pontuados estão relacionados justamente à monitorização e ao controle dos pacientes, bem como ao suporte respiratório e do débito urinário.

Em um estudo realizado na Coréia do Sul, foi evidenciada uma relação direta entre o inadequado dimensionamento de profissionais de enfermagem e o aumento dos eventos adversos. Aponta que existe uma correlação direta entre o maior número de pacientes por enfermeiro com as chances de falhas relacionadas à segurança do paciente, má qualidade dos cuidados e cuidados deixados de lado devido à falta de tempo. ${ }^{14}$

O dimensionamento de pessoal trata-se de um importante recurso para a produtividade e a melhoria dos serviços de enfermagem. Dimensionar a quantidade necessária de trabalhadores 
Carga de trabalho de enfermagem em sala de recuperação pós-anestésica: um estudo misto | 16

para a prestação de serviços de saúde implica na identificação da carga de trabalho existente, relacionada ao quantitativo de pacientes, grau de dependência e tempo médio de assistência. ${ }^{15}$

De acordo com estudo realizado em unidade coronariana, o valor do NAS elevado, demonstra que pacientes em unidades especializadas requerem uma elevada carga de trabalho por parte da equipe de enfermagem, com cuidados e monitorizações específicas, que demandam mais da metade do tempo de um profissional no cuidado direto. ${ }^{16}$

A análise descritiva dos dados mais expressivos, obtidos por meio do NAS neste estudo, evidencia que no item um do instrumento, monitorização e controles, $91 \%$ dos pacientes pontuaram no subitem b, ou seja, os mesmos demandaram de presença à beira do leito e observação ou atividade contínua por duas horas ou mais em algum plantão por razões de segurança, gravidade ou terapia, tais com: ventilação mecânica não invasiva, desmame, agitação, confusão mental, posição prona, procedimentos de doação de órgãos, preparo e administração de fluidos ou medicação, auxílio em procedimentos específicos. Este contexto demonstra a complexidade dos pacientes atendidos na referida instituição, e ainda pode ser identificado nas falas dos trabalhadores. Os depoimentos indicam que a instabilidade do paciente no pósoperatório é o principal fator que eleva a carga de trabalho em SRPA. Além disso, os resultados de um estudo desenvolvido em unidade de recuperação pós-anestésica são convergentes a este, uma vez que $76 \%$ dos pacientes pontuaram no referido subitem do instrumento. ${ }^{17}$

Os pacientes em unidade de recuperação pós-anestésica apresentam especificidades da assistência de enfermagem como monitorização dos parâmetros vitais a cada 15 minutos na primeira hora, monitorização hemodinâmica invasiva, restrição ao leito, devido ao despertar anestésico, administração de medicamentos e procedimentos de higiene e conforto. Essas características elevam o grau de dependência do paciente frente à equipe de enfermagem que, necessita possuir conhecimentos especializados e habilidades para a tomada de decisão rápida para atuar neste contexto. ${ }^{5}$ 
17 | Pinheiro ALU, Cunha QB, Pai Daiane Dal, Silva RM, Lima SBS, Camponogara S

A alta demanda de pacientes cirúrgicos e os poucos leitos de terapia intensiva podem resultar no aumento do quantitativo de pacientes críticos sob cuidados dos trabalhadores da sala de recuperação pós-anestésica. Cabe à equipe de enfermagem um conhecimento específico e especializado de fisiologia, procedimentos cirúrgicos e gerenciamento de potenciais complicações. ${ }^{18}$

Seguindo os domínios do NAS mais evidenciados neste estudo, tem-se as investigações laboratoriais (item dois), a administração de medicamentos (item três), suporte respiratório (item nove) os quais pontuaram, respectivamente, em $63 \%$, 93\% e $68 \%$ das medidas. Além disso, os depoimentos dos participantes corroboram esses achados como atividades que demandam tempo, atenção e que fazem parte da rotina dos trabalhadores. Essas intervenções também foram frequentemente realizadas em outros estudos e populações, obtendo dados igualmente expressivos. ${ }^{10,19}$

As atividades Monitorização e controle, Suporte e cuidados aos familiares e pacientes, Tarefas administrativas e gerenciais e Intervenções específicas fora da SRPA foram pontuados em $100 \%$ dos pacientes, corroborando com os achados de outro estudo. ${ }^{17}$ A elevada pontuação no item suporte e cuidados aos familiares e pacientes refere-se às informações e orientações realizadas pelos enfermeiros aos familiares e pacientes. As intervenções específicas fora da SRPA referem-se a exames diagnósticos, uma vez que, não raramente, os pacientes ali permanecem internados, necessitando de transporte para tais investigações, e ainda, a transferência da cama para a maca, no momento da alta do paciente para a unidade de internação. Mais uma vez, percebe-se a convergência dos achados quantitativos e qualitativos, tendo em vista que os depoimentos também sinalizam que a alta rotatividade de pacientes do setor contribui para o aumento da carga de trabalho da enfermagem, gerando demandas tanto administrativas quanto assistenciais. 
Carga de trabalho de enfermagem em sala de recuperação pós-anestésica: um estudo misto | 18

Nesse sentido, o enfermeiro exerce inúmeras atividades, muitas delas, relacionadas à assistência indireta ao paciente, como as atividades administrativas e gerenciais (item oito), pontuadas de forma expressiva no NAS deste estudo. Essas atividades, privativas do enfermeiro, por vezes, são colocadas em segundo plano, devido situações que os mesmos caracterizam como mais urgentes. Autores afirmam que existe a necessidade de rever o processo de trabalho, de forma que maior tempo seja disponibilizado às atividades profissionais específicas, envolvidas na assistência ao paciente. ${ }^{20}$

O NAS elevado encontrado nesta investigação, convergente com os depoimentos dos trabalhadores de enfermagem, os quais relatam elevada carga de trabalho, vem ao encontro com estudos que abordam questões relacionadas à saúde do trabalhador. De forma geral, o processo de trabalho de enfermagem envolve rotinas desgastantes, as quais exigem habilidades e responsabilidades em sua execução. Estes aspectos influenciam na qualidade de vida do trabalhador de enfermagem. ${ }^{21}$ Ainda, este estudo identificou que o dimensionamento de pessoal é determinante para que a sobrecarga não seja uma constante no trabalho, minimizando o desgaste deste profissional, preservando assim sua saúde.

Os resultados apresentados na presente investigação podem contribuir para a organização do processo de trabalho da equipe de enfermagem da SRPA, principalmente no que se refere ao dimensionamento de pessoal e carga de trabalho demandada por cada paciente. Os enfermeiros, na divisão e organização do trabalho, precisam estar atentos para este fator, minimizando a sobrecarga de trabalho dos profissionais. Estes resultados também buscam contribuir para o crescimento das publicações acerca da temática em estudo. Destaca-se que a utilização do método misto, com a estratégia explanatória sequencial, contribuiu para o aprofundamento na compreensão dessa problemática, considerando que os achados qualitativos ajudaram a aprofundar a compreensão dos resultados quantitativos inicialmente coletados. 
19 | Pinheiro ALU, Cunha QB, Pai Daiane Dal, Silva RM, Lima SBS, Camponogara S

As limitações deste estudo estão relacionadas à escassa produção de estudos que apliquem o NAS em cenário de recuperação anestésica, limitando as comparações. Além disso, destaca-se a dificuldade na utilização do NAS baseada nos registros de enfermagem, tendo em vista que algumas atividades podem ter sido realizadas e não registradas, e desta forma, não pontuadas no instrumento.

Sugere-se a aplicação do instrumento neste cenário em novos estudos, a fim de ampliar o campo de investigação e possibilitar comparações. Frente aos resultados encontrados, faz-se algumas recomendações para assistência de enfermagem: a realização da medida da carga de trabalho rotineiramente, a fim de fazer uma distribuição de pacientes por trabalhador de acordo com a demanda de cuidados, contribuindo para amenizar a sobrecarga de trabalho; e reavaliar o dimensionamento de trabalhadores de enfermagem escalados por turno, baseado na realidade da instituição/unidade estudada.

\section{Conclusão}

O perfil dos pacientes que estiveram internados na SRPA, no período do estudo, foi, predominantemente, do sexo masculino $(55,5 \%)$, os quais, em sua maioria, eram provenientes da Unidade de Clínica Cirúrgica do hospital (38,28\%) seguido do Pronto Socorro $(33,01 \%)$ e demais unidades. Nesta investigação, a carga de trabalho da equipe de enfermagem, analisada quantitativamente por meio do NAS, mostrou-se elevada, uma vez que cada paciente ocupa em média 57\% do tempo de um trabalhador e que, cada profissional de enfermagem presta cuidados a três ou quatro pacientes por turno.

A percepção dos trabalhadores frente à carga de trabalho expressa nas entrevistas contribuiu para melhor compreensão e aprofundamento dos resultados obtidos no instrumento quantitativo. Os resultados dos depoimentos foram organizados em duas categorias: Características dos pacientes e situações determinantes para a carga de trabalho e Organização 
Carga de trabalho de enfermagem em sala de recuperação pós-anestésica: um estudo misto | 20

do trabalho: características que influenciam na carga de trabalho. $\mathrm{Na}$ primeira categoria evidenciou-se que, os pacientes instáveis e que demandam cuidados críticos são os que geram maior carga de trabalho para a equipe de enfermagem. Na segunda categoria, os trabalhadores relatam que a falta de leitos hospitalares, que reduz a rotatividade do setor e assim, faz com que os pacientes permaneçam em regime de internação, influenciam na carga de trabalho e ainda, a necessidade de reavaliação do dimensionamento de pessoal, tendo em vista a evidência de sobrecarga de trabalho.

Houve convergência entre achados quantitativos e qualitativos. Analisando conjuntamente os achados quantitativos e qualitativos, abre-se a possibilidade de, ao identificar a carga de trabalho bem como o tempo de assistência de enfermagem, adequar o quantitativo de profissionais para determinada demanda de cuidados, com condições de trabalho seguras para a equipe e pacientes.

A utilização do instrumento NAS mostrou-se adequada aos objetivos propostos no estudo, bem como ao cenário da realização da pesquisa, uma vez que, devido às especificidades locais, foi possível pontuar as atividades de enfermagem realizadas de forma individual e, assim, mensurar a carga de trabalho de enfermagem mais próxima do real.

\section{Referências}

1. Fundação Oswaldo Cruz. Pesquisa retrata perfil de 1,6 milhão de profissionais de enfermagem no Brasil. Rio de Janeiro; 2015 [acesso em 2015 ago 04]. Disponível em: http://portal.fiocruz.br/pt$\mathrm{br} /$ content/pesquisa-retrata-perfil-de-16-milhao-de-profissionais-de-enfermagem-no-brasil

2. Aiken LH, Sloane DM, Bruyneel L, Van den Heede K, Sermeus W. Nurses' reports of working conditions and hospital quality of care in 12 countries in Europe. Int J Nurs Stud [Internet]. 2013 fev [acesso em 2019 out 18];50(2):143-53. Disponível em: https://www.sciencedirect.com/science/article/abs/pii/S0020748912004105?via\%3Dihub doi: 10.1016/j.ijnurstu.2012.11.009 
3. Dias MCCB. Aplicação do Nursing Activitis Score (NAS) como instrumento de medida de carga de trabalho de enfermagem em UTI cirúrgica cardiológica [dissertação]. São Paulo (SP): Universidade de São Paulo; 2006. 115 p.

4. Queijo AF, Padilha KG. Nursing Activities Score (NAS): adaptação transcultural e validação para a língua portuguesa. Rev Esc Enferm USP [Internet]. 2009 dez [acesso em 2019 out 18];43(N Esp):1018-25. Disponível em: http://www.scielo.br/scielo.php?script=sci_arttext\&pid=S0080-62342009000500004 doi: 10.1590/S0080-62342009000500004

5. Lima LB, Borges D, Costa S, Rabelo ER. Classificação de pacientes segundo o grau de dependência dos cuidados de enfermagem e a gravidade em unidade de recuperação pós-anestésica. Rev Latinoam Enferm [Internet]. 2010 out [acesso em 2018 maio 03];18(5):881-7. Disponível em: http://www.scielo.br/scielo.php?pid=S0104-11692010000500007\&script=sci_arttext\&tlng=pt doi: 10.1590/S0104-11692010000500007

6. Creswell JW, Clark VLP. Pesquisa de métodos mistos. 2ª ed. Porto Alegre (RS): Penso, 2013.

7. Bardin L. Análise de conteúdo. São Paulo: Edições 70; 2011.

8. Machado MH, Aguiar Filho W, Lacerda WF, Oliveira E, Lemos W, Wermelinger M, et al. Características gerais da enfermagem: o perfil sócio demográfico. Enferm Foco [Internet]. 2015 [acesso em 2019 out 18];6(1/4):11-7. Disponível em: http://revista.cofen.gov.br/index.php/enfermagem/article/view/686/0 doi: 10.21675/2357-707X.2016.v7.nESP.686

9. Gonçalves LA, Padilha KG. Fatores associados à carga de trabalho de enfermagem em unidade de terapia intensiva. Rev Esc Enferm USP [Internet]. 2007 [acesso em 2019 out 18];41(4):645-52. Disponível em: http://www.scielo.br/pdf/reeusp/v41n4/14.pdf

10. Timmers TK, Hulstaert PF, Leenen LP. Patient outcomes can be associated with organizational changes. Crit Care Nurs Q [Internet]. 2014 jan-mar [acesso em 2019 out 18];37(1):125-34. Disponível em: https://www.ncbi.nlm.nih.gov/pubmed/24309466 doi: 10.1097/CNQ.0000000000000011

11. Lima LB, Rabelo ER. Carga de trabalho de enfermagem em unidade de recuperação pós-anestésica. Acta Paul Enferm [Internet]. 2013 [acesso em 2019 out 18];26(2):116-22. Disponível em: http://www.scielo.br/scielo.php?script=sci_arttext\&pid=S0103-21002013000200003 doi: 10.1590/S010321002013000200003

12. Chan RPC, Auler Junior JOC. Estudo retrospectivo da incidência de óbitos anestésico-cirúrgicos nas primeiras 24 horas: revisão de 82.641 anestesias. Rev Bras Anestesiol [Internet]. 2002 [acesso 2019 out 18]; 52(6):719-27. Disponível em: http://www.scielo.br/scielo.php?script=sci_abstract\&pid=S003470942002000600009\&lng=en\&nrm=iso\&tlng=pt doi: 10.1590/S0034-70942002000600009

13. Conselho Federal de Enfermagem (BR). Resolução COFEN № 543/2017, de 18 de abril de 2017. Atualiza e estabelece parâmetros para o dimensionamento do quadro de profissionais de enfermagem nos 
Carga de trabalho de enfermagem em sala de recuperação pós-anestésica: um estudo misto | 22

serviços/locais em que são realizadas atividades de enfermagem[Internet]. Brasília (DF): COFEN; 2017 [acesso em 2018 set 13]. Disponível em: http://www.cofen.gov.br/resolucao-cofen-5432017_51440.html

14. Cho E, Lee N-J, Kim E-Y, Kim S, Lee K, Park K-O, et al. Nurse staffing level and overtime associated with patient safety, quality of care, and care left undone in hospitals: a cross-sectional study. Int J Nurs Stud [Internet]. 2016 maio [acesso em 2019 out 18];60:263-71. Disponível em: https://www.sciencedirect.com/science/article/abs/pii/S0020748916300566?via\%3Dihub doi: 10.1016/j.ijnurstu.2016.05.009

15. Tillvitz LR. Dimensionamento de pessoal de enfermagem do centro cirúrgico de um hospital do norte do Paraná [dissertação]. Ribeirão Preto (SP): Universidade de São Paulo, 2013. 205 p.

16. Reich R, Vieira DFVB, Lima LB, Rabelo-Silva ER. Carga de trabalho em unidade coronariana segundo o Nursing Activities Score. Rev Gaúch Enferm [Internet]. 2015 [acesso em 2019 out 18];36(3):28-35. Disponível em: http://www.scielo.br/scielo.php?pid=S1983-14472015000300028\&script=sci_arttext\&tlng=pt doi: 10.1590/1983-1447.2015.03.51367

17. Lima LB. Nursing Activities escore para avaliação da carga de trabalho de enfermagem em unidade de recuperação pós-anestésica [dissertação]. Porto Alegre (RS): Universidade Federal do Rio Grande do Sul; 2010. 62 p.

18. Saraiva EL, Sousa CS. Pacientes críticos na unidade de recuperação pós-anestésica: revisão integrativa. Rev SOBECC [Internet]. 2015 [acesso em 2019 out 18];20(2):104-12. Disponível em: https://revista.sobecc.org.br/sobecc/article/view/10/8

19. Camuci MB, Martins JT, Cardeli A AM, Robazzi MLCC. Nursing Activities Score: nursing work load in a burns Intensive Care Unit. Rev. latinoam. Enferm. [Internet] 2014 Mar./Apr. [acesso em 2019 out 18]; 22(2): 325-331. Disponível em: http://www.scielo.br/scielo.php?script=sci_arttext\&pid=S010411692014000200325 doi: 10.1590/0104-1169.3193.2419

20. Garcia EA, Fugulin FMT. Distribuição do tempo de trabalho das enfermeiras em Unidade de Emergência. Rev Esc Enferm USP [Internet]. 2010 dez [acesso em 2019 out 18];44(4):1032-8. Disponível em: http://www.scielo.br/scielo.php?script=sci_arttext\&pid=S0080-62342010000400025 doi: 10.1590/S008062342010000400025

21. Amaral JF, Ribeiro JP, Paixão DX. Qualidade de vida no trabalho dos profissionais de enfermagem em ambiente hospitalar: uma revisão integrativa. Espaç Saúde [Internet]. 2015 [acesso em 2019 out 18]; 16(1):66-74. Disponível em: http://docplayer.com.br/17821041-Qualidade-de-vida-no-trabalho-dosprofissionais-de-enfermagem-em-ambiente-hospitalar-uma-revisao-integrativa.html

\section{Autor correspondente}

Nome: Ana Lúcia Uberti Pinheiro 
E-mail: aninhaupinheiro@yahoo.com.br

Endreço: Rua Araújo Viana, 545/404. Santa Maria/RS.

CEP: $97015-040$

\section{Contribuições de Autoria}

\section{1 - Ana Lúcia Uberti Pinheiro}

Participou da concepção e planejamento do projeto de pesquisa, obtenção, análise e interpretação dos dados, e redação do manuscrito.

\section{2 - Quézia Boeira da Cunha}

Participou da concepção e planejamento do projeto de pesquisa, obtenção de dados, e redação do manuscrito.

\section{3 - Daiane Dal Pai}

Participou da revisão crítica do manuscrito.

\section{4 - Rosângela Marion da Silva}

Participou da revisão crítica do manuscrito.

\section{5 - Suzinara Beatriz Soares de Lima}

Participou da revisão crítica do manuscrito.

\section{6 - Silviamar Camponogara}

Participou da concepção e planejamento do projeto de pesquisa, análise de dados, e redação do manuscrito.

\section{Como citar este artigo}

Pinheiro ALU, Cunha QB, Pai Daiane Dal, Silva RM, Lima SBS, Camponogara S. Carga de trabalho de enfermagem em sala de recuperação pós-anestésica: um estudo misto. Rev. Enferm. UFSM. 2019 [Acesso em: Anos Mês Dia]; vol.9 e6: 1-23. DOI:https://doi.org/10.5902/2179769240333 